\title{
Eaux glaciaires, transports solides et décantation en hydro-électricité
}

\author{
A. Bezinge R. Aeschlimann \\ Grande Dixence S.A., Sion
}

\section{Généralités}

L'utilisation intensive des torrents d'origine glaciaire pour la production d'énergie hydro-électrique a conduit la société Grande Dixence S.A. à étudier les différents phénomènes de transports solides depuis 1948 à nos jours. L'important aménagement hydro-électrique de Grande Dixence S.A., situé dans les Alpes valaisannes, comprend 35 bassins versants de 1 à $80 \mathrm{~km}^{2}$ de glaciations et d'orientations différentes, avec des micro-climats divers. Les nombreux ouvrages et les mesures faites ont permis d'analyser différents paramètres, et l'exploitation de l'aménagement représente un précieux et vaste champ d'expériences. Les nombreux bassins glaciaires de haute altitude présentent des différences notables de surface, de glaciation, d'orientation, de topographie et de composition géologique.

Les matériaux transportés sont la cause d'usures des ouvrages et des machines hydrauliques et de dépôts parasites dans les bassins d'accumulation. Cette étude pragmatique présente une synthèse des expériences concrètes faites, ainsi que celles obtenues par une prise flottante originale, installée dans un lac avec un marnage de $30 \mathrm{~m}$. En fin d'étude, l'on présente la conception nouvelle d'une prise en rivière avec un dessableur à écoulements transverses, ainsi que les possibles améliorations des dessableurs actuels. Cette étude permet de mieux saisir la diversité des transports solides et leur décantation en régions alpines et ces expériences peuvent s'appliquer à d'autres régions du globe : Cordillères, Indoukouch et Himalaya.

\section{Transports solides}

Les glaciers et les torrents glaciaires érodent les terrains et les matériaux arrachés vont des blocs les plus gros $\left(100 \mathrm{~m}^{3}\right)$ aux grains les plus fins. Ces transports solides sont très abrasifs, surtout en zones de roches cristalline ou métamorphique.

\subsection{Charriages des matériaux roulés ou en saltation}

Les valeurs extrêmes ci-après sont tirées des purges estivales que l'on a exécutées sur des bassins de dégravelage. Ces valeurs sont maximales en années chaudes et lors d'années froides les charriages sont faibles, 40 à $50 \%$ des valeurs moyennes: volumes spécifiques: de 20 à $800 \mathrm{~m}^{3} / \mathrm{km}^{2} / \mathrm{an}$.

La surface des bassins versants ne joue pas un grand rôle, sauf si l'activité du glacier devient importante. Certains bassins glaciaires à forte pente présentent des charriages insignifiants (faibles épaisseurs des glaces et géologie favorable!).

Sur certains bassins, des orages peuvent déclencher des coulées morainiques et de boue de plusieurs milliers de $\mathrm{m}^{3}$ (moraines sur fortes pentes!). Sur un bassin de $5 \mathrm{~km}^{2}$, lors d'une débâcle glaciaire, l'on a retrouvé $70-80000 \mathrm{~m}^{3}$ de matériaux transportés entre 2800 et $1400 \mathrm{~m}$ d'altitude, à la suite d'une vidange lente d'une poche glaciaire de $100000 \mathrm{~m}^{3}$ (débit de pointe : $4-5 \mathrm{~m}^{4} / \mathrm{s}$ !).

\section{Glacial waters, carriage of solid materials and decantation in hydro-electricity}

Grande Dixence S.A., is a large hydro-electric scheme in the mountains. It was built and operated since 1953-1965: 27 sand and gravel traps of the GD-EdF type, 10 gravel traps, 11 classical sand traps.

The study presents a synthesis of experiences done in this field as well as those made on an original floating water intake of a compensating basin with a water-level variation of $30 \mathrm{~m}$.

Finally, it is presented the actually conception of a river water intake and sandtrap with a transversal through flow the possible future ameliorations on sand traps. 


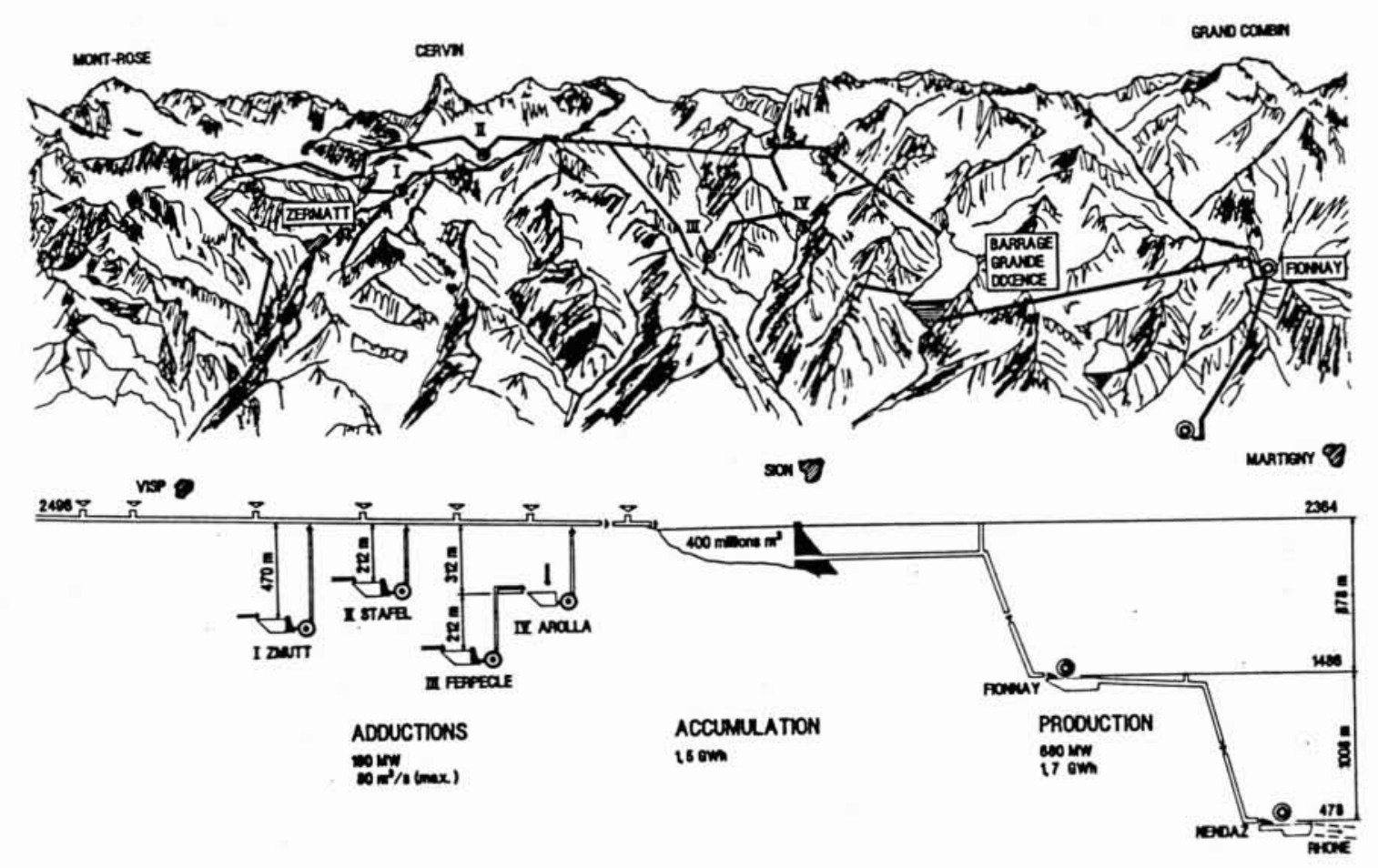

1. Aménagement de Grande Dixence. Situation et profil en long.

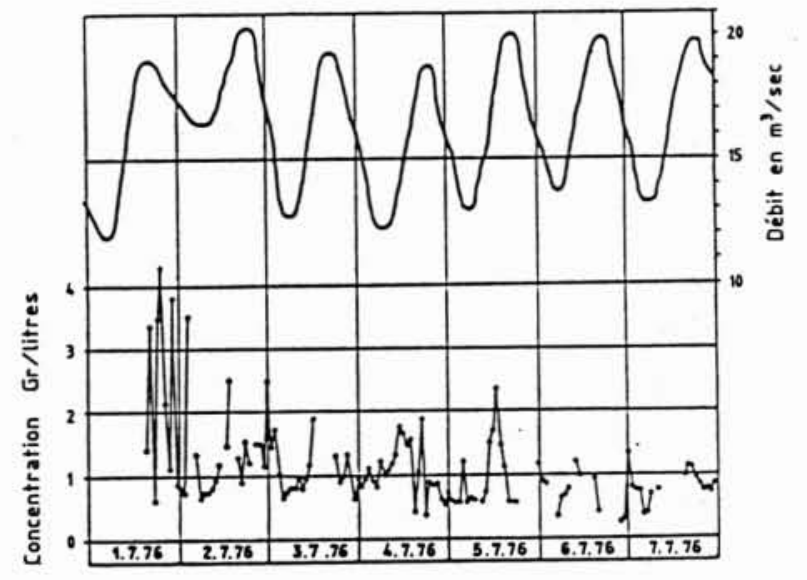

2. Torrent de Gorner. Débit et concentrations (mesures Collins).

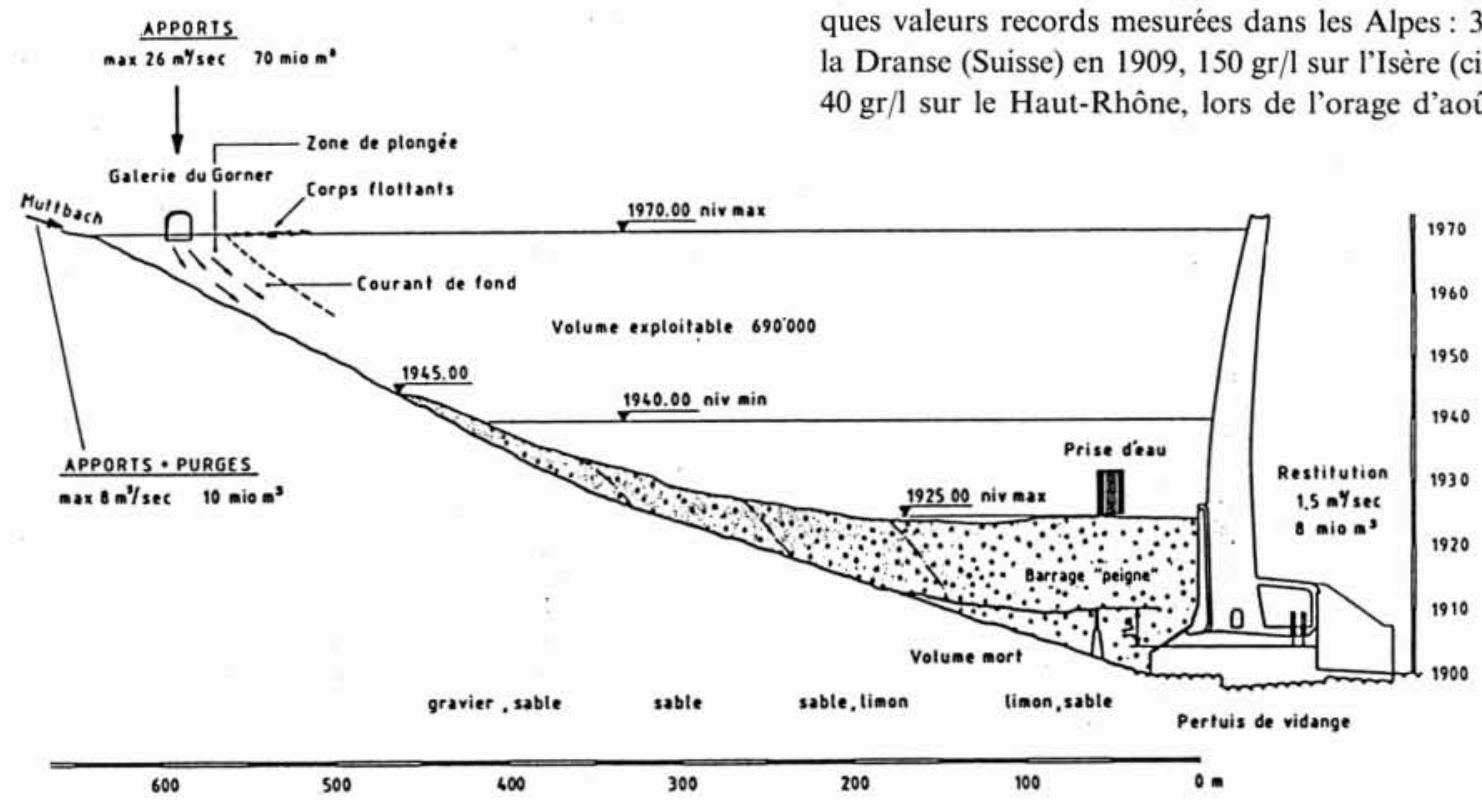

3. Bassin de Z'Mutt. Profil en long.
On peut affirmer que les grands cônes de déjection que l'on rencontre dans les vallées alpines proviennent de phénomènes extrêmes: orages intenses, débâcles glaciaires importantes, etc.

\subsection{Matériaux en suspension (fig. 2)}

Ces matériaux fins proviennent essentiellement du frottement du glacier sur son lit rocheux et de l'abrasion élevée causée par l'écoulement torrentiel des eaux glaciaires. La détermination des concentrations et la granulométrie des matériaux transportés intéressent les sociétés hydroélectriques, car elles permettent de déterminer les risques et l'importance des dépôts et des usures possibles.

Pour les étés (1973 chaud avec gros débit et 1974 froid avec faible débit), l'on a mesuré respectivement :

Bassin de Gorner $\quad 80 \mathrm{~km}^{2} \quad 800$ et $450 \mathrm{~m}^{3} / \mathrm{km}^{2}$ Bassin de Tsidjiore $\quad 6 \mathrm{~km}^{2} 500$ et $120 \mathrm{~m}^{3} / \mathrm{km}^{2}$ avec des concentrations records de $15-20 \mathrm{gr} / 1$.

Il faut relever la grande variabilité des concentrations sans corrélation bien marquée avec les débits. Voici quelques valeurs records mesurées dans les Alpes : $36 \mathrm{gr} / 1$ sur la Dranse (Suisse) en 1909, $150 \mathrm{gr} / \mathrm{l}$ sur l'Isère (cit. Pardé), 40 gr/l sur le Haut-Rhône, lors de l'orage d'août 1987. 


\subsection{Bilan des transports solides}

Après plusieurs années de captages, il a été posible de dresser un inventaire des purges sur les différents bassins du val d'Hérens. Il en résulte les valeurs moyennes des volumes des matériaux charriés et des sédiments transportés (jusqu'à $200 \mu \mathrm{m}$ ). L'on estime la fourchette normale entre volumes min. et max. à $\pm 50 \%$.

Pour des bassins de 2 à $36 \mathrm{~km}^{2}$, avec des glaciations de 20 à $70 \%$, l'on obtient une fourchette des volumes moyens annuels de matériaux de 150 à $1000 \mathrm{~m}^{3} / \mathrm{km}^{2}$.

Lorsque l'on compare les volumes spécifiques des matériaux transportés par suspension avec les matériaux transportés par charriage, on relève: pour Stafel et Ferpècle $\left(36 \mathrm{~km}^{2}\right.$ : glaciers moyens, env. $\left.20 \mathrm{~km}^{2}\right)$, des volumes semblables ; pour Gorner $\left(80 \mathrm{~km}^{2}\right.$ : grand glacier, env. 60 $\mathrm{km}^{2}$ ), les matériaux fins sont trois à quatre fois plus importants que les graviers.

\subsection{Matériaux en suspension dans des lacs avec eaux glaciaires (fig. 3)}

Dans le lac de Z'Mutt, $800000 \mathrm{~m}^{3}$, long de $800 \mathrm{~m}$ et profond de $70 \mathrm{~m}$, les concentrations de fines en suspension ont été mesurées sur un profil vertical au droit de la prise d'eau.

Avec des pièges à sédiments placés à différentes profondeurs du lac, (mesures sur plusieurs jours) : les concentrations sont mesurées de façon relative, mais elles intègrent bien les concentrations moyennes à différentes profondeurs. Elles donnent des concentrations de fond 4 à 7 fois plus élevées qu'en surface. Malgré cela, les concentrations de fond sont encore deux fois plus faibles que les eaux d'arrivée (en queue de retenue), ce qui met en évidence un phénomène de dilution partielle. En analysant le fines récupérées, on s'aperçoit que les courbes granulométriques varient fortement. Ainsi, tous les grains supérieurs à $40 \mu \mathrm{m}$ (plus de $60 \%$ ) qui s'écoulent dans le fond du lac ne se retrouvent plus $30 \mathrm{~m}$ plus haut.

Lors d'un soutirage permanent par pompage, il se crée un courant de densité à partir d'un débit d'arrivée de $11-12 \mathrm{~m}^{3} / \mathrm{sec}$. Par recoupements, ce courant de densité s'écoule à une vitesse de $0,40-0,50 \mathrm{~m} / \mathrm{s}$.

L'analyse du détail des particules en suspension montre, en majeure partie, des grains de silices, aux surfaces très rugueuses, aux aspects brisés et éclatés et de formes généralement lamellaires (microscope électronique), ce qui favorise leur suspension dans un liquide visqueux.

Du fait des eaux à basses températures $\left(1-2^{\circ} \mathrm{C}\right)$, la viscosité cinématique est élevée. Ainsi, ces particules soumises à des forces importantes de viscosité ne se décantent que très lentement (forces gravitaires faibles). Ainsi, tous les lacs glaciaires sont troubles et de couleur différente, déterminée par la géologie.

Comme bassin de décantation, le lac de Z'Mutt, long et profond, n'est efficace que sur 10 à $15 \%$ des concentrations, dans toute la gamme de granulométrie.

Pour le lac de Grande Dixence, contenance $400 \mathrm{mio} \mathrm{m}^{3}$, profondeur maximum $200 \mathrm{~m}$, le phénomène de sédimen- tation est très efficace. Ceci provient surtout de l'arrivée des eaux brutes à l'extrémité amont du lac, tandis que le soutirage de l'eau se fait au barrage, $5,5 \mathrm{~km}$ en aval.

Pour l'été 1975, année hydrologique moyenne, on a mesuré une concentration moyenne de $0,4 \mathrm{gr} / 1$ sur l'arrivée des eaux. Compte tenu des $350 \mathrm{mio} \mathrm{m}^{3}$ d'eau, ce sont environ $140000 \mathrm{~T}$ de sédiments qui sont arrivés dans le lac, soit environ un volume de $90000 \mathrm{~m}^{3}$. Les importants soutirages d'eau ne se produisent qu'en hiver et l'on a mesuré des concentrations de $0,004-0,01 \mathrm{gr} / 1$ sur ces eaux turbinées, soit un très faible volume de matériaux soutirés du lac.

A titre documentaire, la retenue de Mauvoisin en Valais, Suisse, $\left(200 \mathrm{mio} \mathrm{m}^{3}\right)$ a été vidée totalement en 1985 (bassin versant : env. $220 \mathrm{~km}^{2}$, glaciation : env. $25 \%$. Après 30 ans d'exploitation, l'on a mesuré : 6 mio de $\mathrm{m}^{3}$ de dépôts, soit environ 1.5-2 mio de $\mathrm{m}^{3}$ de graviers en queue de retenue et 4-4.5 mio de $\mathrm{m}^{3}$ de sédiments en fond de retenue.

\section{Ouvrages de captages particuliers et expériences}

\subsection{Captage des eaux du glacier du Gorner}

(Bassin versant: $80 \mathrm{~km}^{2}, 2^{\mathrm{e}}$ glacier d'Europe $\sim 60 \mathrm{~km}^{2}$ ).

La rivière glaciaire de Gornera comporte des débits importants et favorise de gros transports solides sous toutes les formes: sédiments, graviers et gros blocs rocheux.

Débits maxima du torrent

en juillet et août : $\quad 30-35 \mathrm{~m}^{3} / \mathrm{s}$

Débits de crue: de $80-200 \mathrm{~m}^{3} / \mathrm{s}$

qui peuvent être atteints lors de la vidange annuelle d'un lac glaciaire de confluence (1-6 mio $\left.\mathrm{m}^{3}\right)$, sous forme d'une débâcle.

Comme les eaux captées alimentent une station de pompage, tous les ouvrages ont été conçus dès l'origine pour diminuer les usures des pompes.

Ouvrages en série, dimensionnés pour un débit nominal de $26 \mathrm{~m}^{3} / \mathrm{s}$

a) Prise en rivière avec un bassin de retenue des gros matériaux.

b) Dégraveleur original pour accumuler $800 \mathrm{~m}^{3}$.

c) Dessableurs classiques avec fond trapézoïdal, disposés en parallèle et dimensionnés chacun pour $13 \mathrm{~m}^{3} / \mathrm{s}$, longueur : $50 \mathrm{~m}$, profondeur et largeur : $8 \mathrm{~m}$.

d) Bassin de compensation avec un volume utile de $600000 \mathrm{~m}^{3}$, complété en 1985 par une prise flottante conçue et créée par GD.

Ces ouvrages sont décrits par la suite.

\subsubsection{Prise d'eau du Gorner (fig. 4, p. suiv.)}

Sur faible pente, implantation d'un barrage en rivière avec :

-1 bassin amont de décantation $\left(800 \mathrm{~m}^{3}\right)$ pour gros matériaux ; 


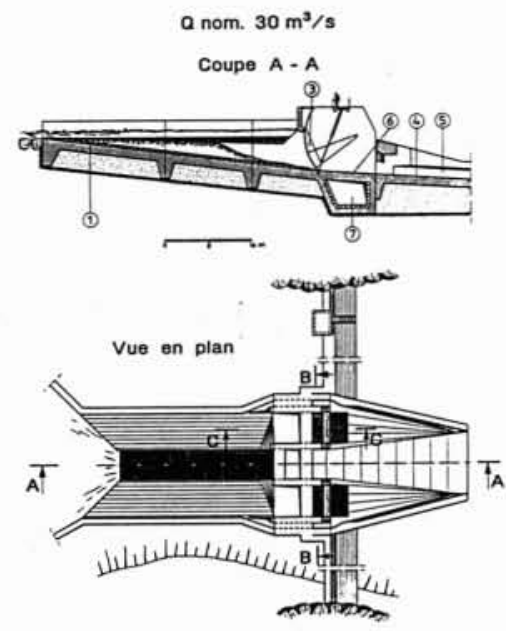

4. Prise de Gorner. Vue en plan et coupe.

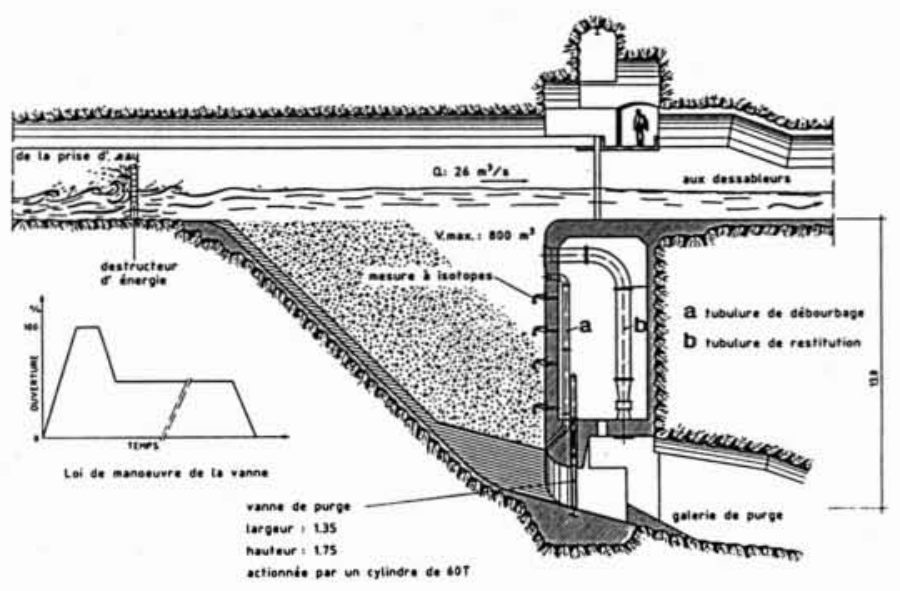

5. Dégraveleur de Gorner. Coupe.

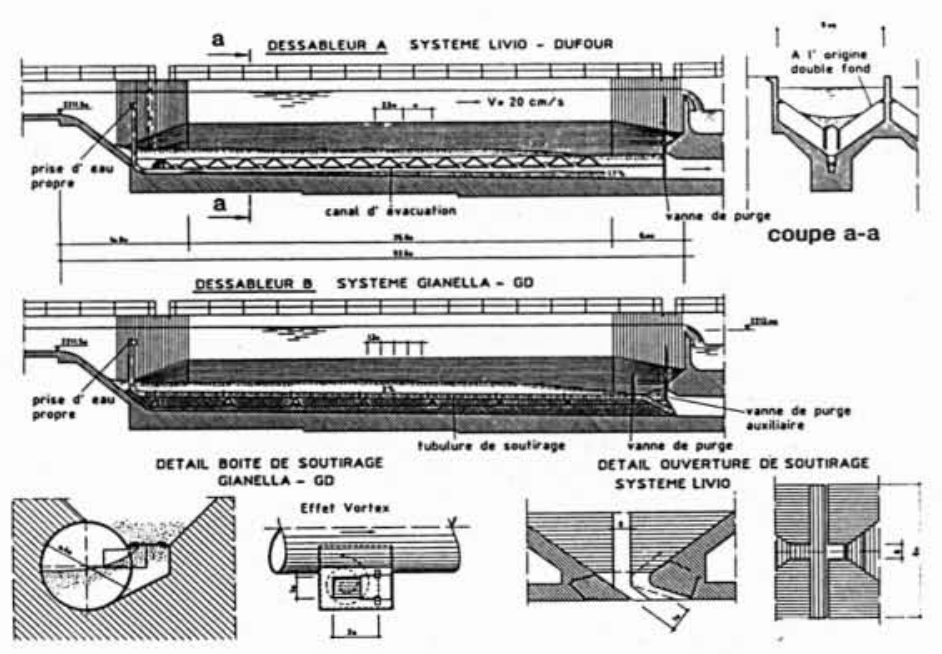

6. Dessableurs de Stafel. Coupes diverses.
- 2 prises latérales, type par en dessous ;

-2 murs bajoyers qui forment déversoir à partir de $30 \mathrm{~m}^{3} / \mathrm{s}$;

- 1 passe centrale fermée par 1 vanne-secteur ;

- détection des niveaux de dépôts par sources radioisotopes avec téléalarmes ;

- télécommande des purges par ouverture de la vanne secteur.

Expériences: Retenue efficace des gros matériaux jusqu'aux sables.

Les problèmes de chasse des matériaux ont été résolus comme suit :

- matériaux ségrégationnés par les grilles (pierres et glace) évacués par l'ouverture sporadique de vannettes latérales avec une déviation appropriée des jets ;

- matériaux déposés dans le bassin principal éliminés par ouverture totale de la vanne secteur de la passe centrale.

Remarque: Lors de la vidange du lac glaciaire avec déversements sur les murs latéraux, des blocs de glaces de plusieurs tonnes, en flottaison, ont détruit la passerelle d'accès, d'où un rehaussement de celle-ci et du local de commande.

Après 20 ans d'exploitation, les expériences faites avec ce captage original sont très satisfaisantes, sans pertes d'eau et avec le minimum d'usures des parties vives.

\subsubsection{Dégraveleur du Gorner (fig. 5)}

But : accumuler $800 \mathrm{~m}^{3}$ de graviers et de sable dans une trémie pour les éliminer rapidement, avec une perte minimale.

Conception: ouvrage conçu par A. BEZINGE et exécuté après des essais sur modèle réduit à l'échelle $1 / 20$. C'est un silo dont la forme a été extrapolée des premiers camions à béton (1960). L'ouvrage, construit en rocher, est profond d'environ $15 \mathrm{~m}$. Des niveaux d'engravement sont détectés par des sources ponctuelles radio-isotopiques de $5 \mathrm{miC}$, permettent de donner des alarmes ou des ordres de purges. La vanne de purge avec un tablier massif et couteau en acier au manganèse est commandée par un vérin de $60 \mathrm{t}$. Elle peut cisailler, si besoin est, des blocs de gneiss de $30 \times 30 \mathrm{~cm}$. Deux tuyaux by-pass conduisent de l'eau sur l'amont du pertuis pour éviter tout effet indésirable de voûte dans le matériel "tassé " et retenu dans le silo. Un groupe de secours, en énergie électrique, est toujours prêt à intervenir.

Expériences: Elles sont très satisfaisantes! A chaque purge, $700-800 \mathrm{~m}^{3}$ de matériaux sont évacués en 1215 minutes. Quelques obstructions accidentelles de la galerie de purge ont disparu par modifications de la loi de purge. Les fortes usures des glissières ont été maîtrisées par une forme convergente du pertuis amont.

Note: Les expériences faites sur modèle réduit et sur l'ouvrage original ont servi de référence pour construire un dégraveleur semblable, avec purge latérale, sur l'aménagement du Fieschertal (Valais, en Suisse). 


\subsection{Dessableurs}

10 dessableurs classiques équipent les ouvrages GD, dimensionnés pour des débits unitaires de 7,5 à $15 \mathrm{~m}^{3} / \mathrm{s}$. Conçus et construits avec des formes traditionnelles (Dufour, Buchi), ils comprennent :

- un bassin allongé avec fond triangulaire et piédroits verticaux, dimensionnés pour des vitesses moyennes d'écoulements de $20 \mathrm{~cm} / \mathrm{s}$. D'après les calculs de l'époque, l'on devait arriver à décanter des grains jusqu'à $200 \mu \mathrm{m}$; - soutirage des sables selon 3 systèmes : 1 dessableur avec Livio-Dufour, 3 dessableurs avec boîtes à vortex GD, 6 dessableurs avec Bieri (décrits par la suite).

Sur les 8 dessableurs qui équipent les installations de pompage, les difficultés suivantes ont été relevées :

- la pénétration des graviers rend difficile l'évacuation des matériaux et de ce fait les dessableurs sont vite saturés et ils ne fonctionnent plus correctement! Les bassins de compensation s'ensablent rapidement. Aussi, sur les machines hydrauliques, les usures deviennent importantes avec comme corollaires : arrêt des machines, coûts de réparations élevés, baisse des performances (pertes économiques) ;

- manœuvres difficiles des vannes à commandes manuelles.

\subsubsection{Dessableurs de Stafel (fig. 6)}

Il s'agit de 2 dessableurs classiques en parallèle avec, $a$ l'origine, un fond intermédiaire, pour obtenir une hauteur réduite de décantation : système étudié en son temps par Labaye (EdF) et Sogreah, et qui sont équipés d'éléments de soutirage Livio-Dufour (dessableur A) ou de tube de soutirage avec boîtes à vortex (dessableur B). Débit : $2 \times 7,5 \mathrm{~m}^{3} / \mathrm{s}$.

\section{Expériences et modifications}

Obstructions nombreuses par les sables et les fines !

Lors des premières vidanges totales des dessableurs, effondrements des fonds intermédiaires par surcharge des dépôts de sables non évacués: section insuffisante des cheminées d'évacuation des sables!

Dessableur A. Suppression du fond intermédiaire en bois et écartement des éléments triangulaires de soutirage. En amont : pose d'un tube d'alimentation du canal de purge en eau de purge. Purges effectuées à intervalles réguliers (horloge) et par ouverture d'une vanne. Résultat: bon soutirage des sables.

Dessableur B. Suppression du fond intermédiaire et, après essais sur modèle réduit, installation d'un système de soutirage, par une tubulure avec des boîtes de sucion par effet " vortex » dont les ouvertures de grandes dimensions : $20 \times 10 \mathrm{~cm}$, sont réglables.

Lors de l'ouverture de la vanne de purge, il se produit d'abord dans la tubulure un écoulement amont-aval avec érosion des sables déposés à l'intérieur, puis cet écoulement produit un effet "vortex " dans les boîtes avec soutirage des sables. La vanne de purge est commandée par une horloge avec séquences réglables.

Résultat : excellent fonctionnement de cette installation simple et bon marché.

Une installation semblable équipe les 2 dessableurs de Findelen (GD). Sur le dessableur unique du Fieschertal, dimensionné pour $15 \mathrm{~m}^{3} / \mathrm{s}$, un système semblable, mais de grandes dimensions, donne toute satisfaction.

Note: Au vu d'incertitudes sur des dépôts résiduels (talus sous eau!), on va installer, sur la zone amont de chaque dessableur, 2 détecteurs d'ensablement à tiges vibrantes, $330 \mathrm{~Hz}$ avec alarmes aux usines de pompage. Essais concluants exécutés sur un bassin de prise!

Efficacité des ouvrages de captage de Stafel et de Gorner sur la décantation

La figure 7 montre les droites de régression des concentrations de sédiments en fonction des débits observés sur les différents ouvrages : prise (eaux brutes), sorties dessableurs (eaux dessablées), pompes (eaux décantées).

La figure 12 montre les courbes granulométriques des sédiments retrouvés en aval des ouvrages de Gorner.

Critique des résultats. Ils montrent que:

- les dessableurs ont une efficacité d'environ $38 \%$;

- le bassin de compensation a une efficacité de $34 \%$;

- les eaux pompées contiennent le solde, soit $\sim 28 \%$.

Avec les nombreux ouvrages de Gorner, construits en série, il s'avère que même avec l'important lac de Z'Mutt, les efficacités sont restreintes. Sur ce lac, la création de courants turbides "de densité » ne favorise pas les décantations.

Comme les concentrations et la granulométrie, influencent les usures des machines hydrauliques, cela nous a conduits à entreprendre les recherches décrites ci-après, soit :

- défauts des dessableurs traditionnels;

- prise avec dessableur transverse ;

- création d'une prise flottante.

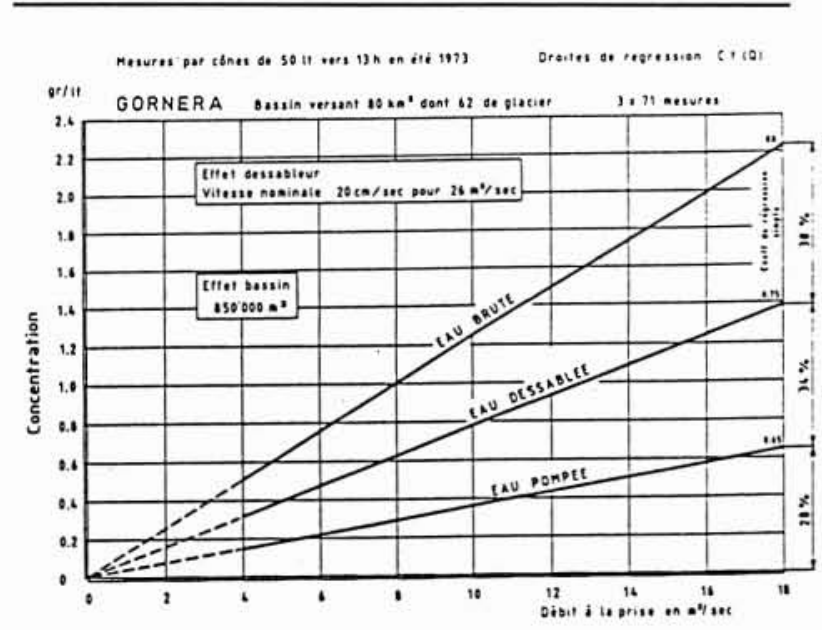

7. Gorner. Efficacité des ouvrages sur les décantations. 


\subsubsection{Défauts du dessableur traditionnel (conception et principe)}

Dépôts importants des matériaux à grosse granulométrie à l'entrée du dessableur, favorisés par les habituelles grilles de tranquillisation. Dans cette zone, évacuation difficile des matériaux.

Trajectoires inattendues des particules fines du fait de l'introduction des eaux turbides dans le haut du dessableur.

Mauvaise décantation, qui ne concorde pas du tout avec les théories, du fait que les particules ont des formes quelconques (coquilles) et du fait que la viscosité de l'eau est maximale $\left(2-4^{\circ} \mathrm{C}\right)$, d'où prédominance des effets de "portances". Gravité inopérante!

Les dessableurs de GD sont trop profonds, d'où des trajectoires trop longues, ce qui favorise la reprise des " fines " aux abords du déversoir frontal (remise en vitesse !).

Répartition aléatoire et turbulente des vitesses, avec quelquefois, proche des parois et en surface, des courants inverses (piédroits en béton ou en rocher!).

\subsection{Dégraveleur-dessableur (fig. 8)}

En haute montagne, pour les captages par gravité, les eaux sont dérivées par des prises par "en dessous " et fosses avec grilles en plafond et les eaux brutes sont conduites dans un bassin dégraveleur-dessableur qui élimine les graviers, sables et fines jusqu'à $300 \mu \mathrm{m}$. Puis, les eaux sont conduites dans le lac d'accumulation par un collecteur.

Ce type de dégraveleur-dessableur est d'origine EdF (Roselend). Il a été modifié et adapté par GD dès 1958. Cet ouvrage, sans courant électrique, permet le contrôle des dépôts de matériaux et permet leurs évacuations automatiques avec l'énergie hydraulique locale.

Sur l'ensemble de l'aménagement de Grande Dixence, 21 dégraveleurs-dessableurs de ce type fonctionnent convenablement avec ce principe autonome. Certains comportent des bassins de 1500 à $2000 \mathrm{~m}^{3}$ équipés pour des débits nominaux de $8-10 \mathrm{~m}^{3} / \mathrm{s}$, soit des débits maxima de purge de $15-18 \mathrm{~m}^{3} / \mathrm{s}$. Certains exécutent $50-60$ purges par été.

\section{Conception du dégraveleur-dessableur}

Bassin de section trapézoïdale à forte pente, (min. $8 \%$ ) avec section croissante pour ralentir les écoulements avec une vitesse de $20 \mathrm{~cm} / \mathrm{s}$ au débit nominal et au $2 / 3$ de la longueur. Cette vitesse permet, avec les viscosités en jeu, de décanter des sédiments de $300 \mu \mathrm{m}$ environ.

Le dépôt des matériaux se fait sous forme d'un talus alluvionnaire avec classification des matériaux de gros à fin entre l'entrée et la vanne de purge.

Par ouverture de la vanne de purge, il se produit une vidange totale du dégraveleur avec une évacuation complète des matériaux déposés (écoulements torrentiels intenses) et la vanne se referme automatiquement après 20 à 30 minutes de purge.
Expériences générales réalisées sur l'ensemble des dégraveleurs-dessableurs

La sédimentation est efficace tant que l'on ne laisse pas se former un dépôt important (grains max. après déversement : $300 \mu \mathrm{m}$ !

L'autonomie du système automatique de palpage et de purge, avec l'énergie hydraulique, demande beaucoup de soins: nettoyage régulier des crépines, des horloges hydrauliques et des bassins (dépôts localisés !), contrôle des fixations et de l'alignement des poulies, pose de câbles en acier inoxydable avec tressage antigiratoire.

Quelques difficultés d'exploitation sont à relever: obstruction de l'entrée du dégraveleur avec mise en pression de la galerie d'adduction (lors de coulées morainiques ou d'éboulements glaciaires), fermeture défectueuse de la vanne secteur (pierre coincée).

3.4. Prise avec dessableur-transverse : nouvelle conception (fig. 9)

But : Capter $8 \mathrm{~m}^{3} / \mathrm{s}$ sur une rivière alpine avec un débit de crue qui peut atteindre $150 \mathrm{~m}^{3} / \mathrm{s}$, et dessabler au mieux les eaux dérivées pour les turbiner dans une usine hydroélectrique.

\section{Conception}

Création d'un bassin de retenue en rivière, exploité comme ouvrage dégraveleur à niveau constant jusqu'à un débit dans la rivière de $30-40 \mathrm{~m}^{3} / \mathrm{s}$.

Prélèvement latéral des eaux dérivées par une longue prise noyée et placée si possible à grande hauteur du radier, mais encore à l'abri du gel.

Passage des eaux à travers un dessableur double accolé au bassin. Dessablage par écoulements transverses à basses vitesses et déversoir latéral sur la galerie d'amenée.

Mise hors service de la prise dès un débit de $40 \mathrm{~m}^{3} / \mathrm{s}$ en rivière, par levage de la vanne secteur installée sur le bassin de retenue.

\section{Justifications hydrauliques du dessableur-transverse}

L'alimentation latérale du dessableur par une prise basse et noyée, longue et de grande section, permet l'injection de l'eau à faibles vitesses (soit le $10-15 \%$ des vitesses d'alimentation des dessableurs classiques !), donc faibles soutirages des sédiments du bassin de prise en rivière.

L'injection de l'eau brute à mi-hauteur du dessableur raccourcit les trajectoires de décantation des particules (voir l'application de ce principe sur le dessableur conçu vers 1920 par le Prof. Buchi).

Le profil transversal des vitesses moyennes montre une grande décélération de l'eau à l'entrée du dessableur, d'où une chute « rapide " des grosses particules, $80-200 \mu \mathrm{m}$, des écoulements avec instabilités locales "forcées" dans la zone de décélération, avec utilisation efficace du phénomène par une installation appropriée décrite ci-après (pièges à particules !). 
La disposition adéquate d'une grille permet d'utiliser les instabilités (tourbillons internes!), pour obtenir des précipitations "forcées" de grains derrière un obstacle. La disposition inclinée de fers profilés en $\mathrm{U}$ favorise la collecte des grains pour les conduire vers la zone basse du dessableur. (Expériences de LABAYE, ing. EdF, 1952, et d'après des études faites sur les barrières à neige).

L'installation d'un déversoir latéral, long de $45 \mathrm{~m}$, avec une hauteur de veine déversante de $22 \mathrm{~cm}$ (débit nominal $8 \mathrm{~m}^{3} / \mathrm{s}$ ), permet d'obtenir des faibles vitesses de déversement. Ainsi, la vitesse moyenne de la veine déversante est réduite de $50 \%$ de celle obtenue sur un déversoir frontal (dessableur classique!). Ceci diminue fortement la « reprise » des grains encore en suspension proches du déversoir. (Expériences : EdF et mesures Wilhelm sur le dessableur de Fara à Grande Dixence, 1960).

\section{Remarques}

Certains phénomènes décrits ci-dessus (couches limites) ont été utilisés dans le dessableur conçu par Sogreah (Grenoble) et par Juillard sur l'ouvrage de Trift (Berne, Suisse).

Les séparateurs à lamelles ondulées utilisés pour la classification des boues mettent en jeu les mêmes principes.

Des dessableurs un peu semblables sont utilisés par Degrémont (dessablage des eaux usées) et par l'industrie chimique.

Par rapport à une disposition classique, la conception originale de cet ouvrage prise-dessableur permet, à volume égal des travaux:

- une construction plus rationnelle et une implantation plus facile en vallée alpestre, d'où un moindre coût de construction ;

- une efficacité plus grande sur la décantation des particules, d'où moins d'usures sur les machines hydrauliques ; — l'élimination des grains supérieurs à $100 \mu \mathrm{m}$, malgré la viscosité maximale des eaux à traiter $\left(4^{\circ} \mathrm{C}\right)$.

\subsection{Prise flottante (fig. 3 et 10)}

\section{Constatation}

La station de pompage de Z'Mutt comprend 2 pompes principales : Hmano : $500 \mathrm{~m} \mathrm{CE}, 2$ étages, $\mathrm{n}: 1500 \mathrm{t} / \mathrm{min}$., $\mathrm{Q}: 2 \times 5,5 \mathrm{~m}^{3} / \mathrm{s}$. Ces pompes refoulent les eaux qui proviennent du captage de Gorner. Malgré tous les ouvrages de décantation implantés en série et décrits ci-avant, ces pompes doivent être entièrement réparées et révisées à grands frais chaque hiver.

\section{Démarches et déductions}

Le Prof. T. Bovet a démontré que les usures des machines hydrauliques sont proportionnelles, d'une part au poids total des matériaux solides en suspension dans les eaux transitées, et d'autre part au poids unitaire des particules (puissance 3 de la dimension).

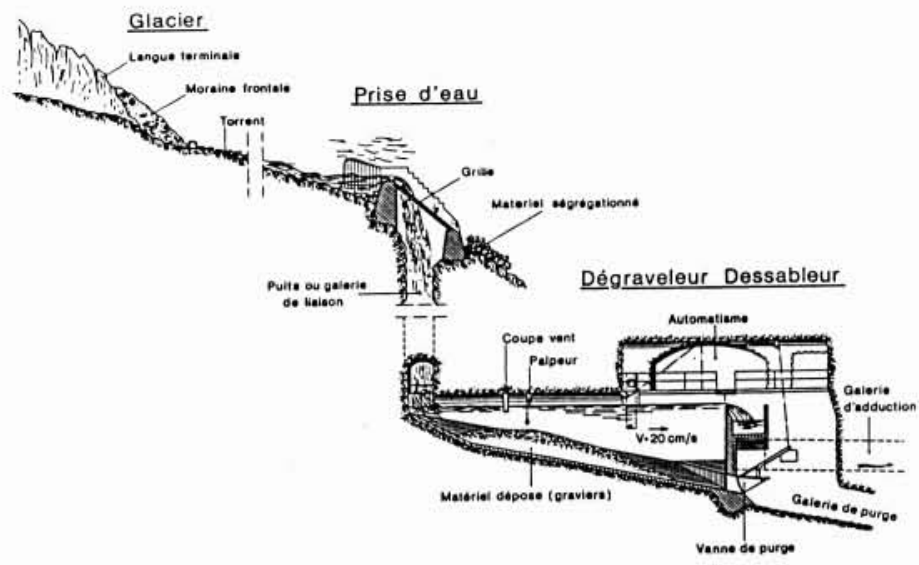

8. Disposition des ouvrages de captage avec dégraveleurdessableur.
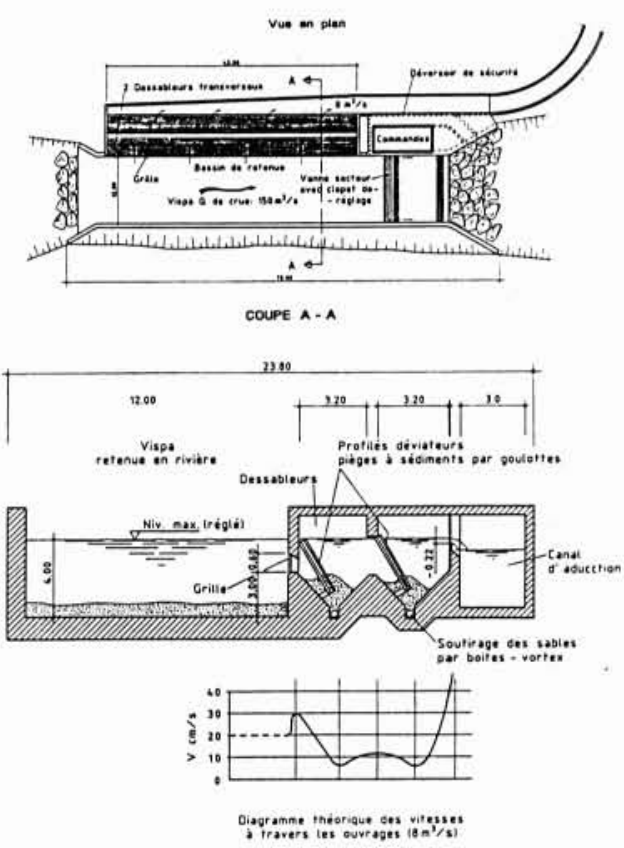

9. Prise avec dessableur-transverse. Vue en plan. Coupe en travers.

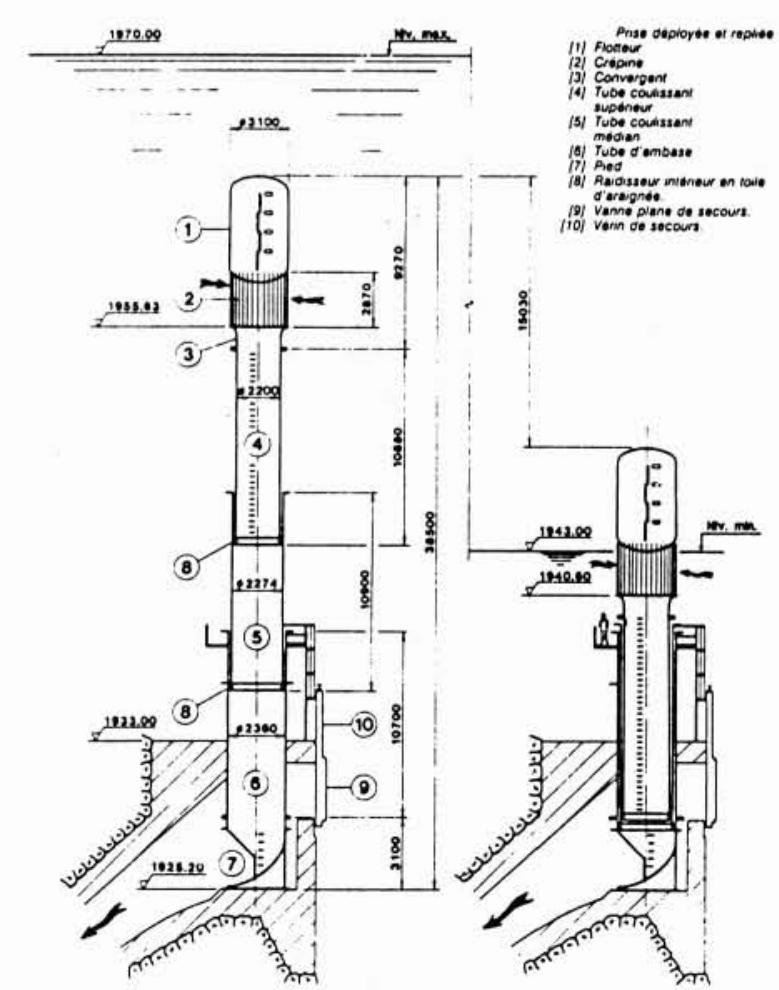

10. Prise flottante déployée et repliée 
Les sédiments prélevés (pièges à sédiments) dans le bassin de Z'Mutt, niveau max. 1970, montrent qu'en passant du niveau 1925 (altitude de la prise d'eau) au niveau 1955, la concentration est sept fois inférieures lors des faibles débits et quatre fois lors des gros débits, tandis que la dimension maximale du $90 \%$ des grains passe de $150 \mu \mathrm{m}$ à $90 \mu \mathrm{m}$.

Dans le cas de Z'Mutt, le traitement des valeurs moyennes de ces paramètres donne un rapport de puissance d'érosion de 1 à 50 entre les eaux captées au niveau max. et celles captées au niveau min. Connaissant l'importance de la dilution et de la granulométrie sur les usures, il devenait logique de vouloir réaliser une installation qui, en variant de hauteur, capterait en tout temps les eaux proches de la surface.

\subsubsection{Conception}

La prise flottante est constituée d'une crépine cylindrique qu'un tube télescopique en trois parties relie à l'ancienne prise. Un flotteur surmonte l'ensemble. Elle est conçue pour suivre, dans certaines limites, les variations du plan d'eau. Sa course a été limitée à $15 \mathrm{~m}$, car il n'était pas rentable d'augmenter au-delà la hauteur de l'installation.

L'ancienne prise est devenue caduque par l'obturation de ses passes. Ses infrastructures sont utilisées comme massif d'ancrage de la nouvelle installation.

Le volume utile du lac reste pratiquement le même, soit environ $625000 \mathrm{~m}^{3}$. En hiver, la prise est bloquée dans sa position basse et noyée pour la protéger des avalanches et de la banquise.

\subsubsection{Essais sur modèle réduit}

Afin de réduire au minimum les inconnues, GD a fait construire dans son usine de Z'Mutt, un modèle réduit à l'échelle 1: 15 comprenant une prise flottante, du type décrit ci-dessus, installée dans une cuve métallique représentant la retenue. En conclusion, ces essais ont permis :

— de constater la validité du concept par l'observation du bon fonctionnement du modèle à tous les niveaux et à tous les régimes ainsi que sa bonne tenue aux vagues et courants latéraux ;

— d'améliorer quelques dispositions constructives et fixer les tolérances d'exécution.

\subsubsection{Construction et montage}

La solution en acier inoxydable présentée par les Ateliers de constructions mécaniques de Vevey a été choisie pour les raisons suivantes :

- la nature du matériau principal, l'acier inoxydable, et le choix de guidages en bronze assurant de bonnes garanties de fonctionnement et de durabilité ;

- l'apport de solutions originales à certaines dispositions constructives.

Après fabrication, un essai de coulissement en position verticale des tubes emboîtés a été effectué en atelier. Les éléments de la prise ont été mis en place à l'aide d'un blondin d'une capacité de $15 \mathrm{t}$ et d'une portée de $200 \mathrm{~m}$ franchissant la vallée.

\subsubsection{Efficacité et conclusions}

Après 4 ans d'exploitation avec des années hydrologiques très fortes, soit des transports solides très importants il s'est avéré que les diminutions d'usures sont estimées à près de $70 \%$ et cette nouvelle installation sera rapidement amortie.

\subsubsection{Projet d'une prise flottante pour Gebidem}

Retenue de Gebidem, 8 mio de $\mathrm{m}^{3}$, eaux du glacier d'Aletsch, chute de $800 \mathrm{~m}$ avec turbines Pelton multijets de 140 MW.

Actuellement, pour l'aménagement de Gebidem-Massa, l'on a en projet l'installation d'une prise semblable dimensionnée pour un débit nominal de $55 \mathrm{~m}^{3} / \mathrm{s}$ et qui pourrait apporter des diminutions d'usures aussi élevées que celles constatées à Z'Mutt.

Note: Ces ouvrages originaux, dont l'idée revient à $\mathrm{A}$. BEzINGE, ont fait l'objet d'un brevet délivré à Grande Dixence S.A.

\section{Dessableurs du futur (fig. 11)}

Par application partielle des quelques principes énoncés, le dessableur à écoulements transverses représente déjà une solution du futur.
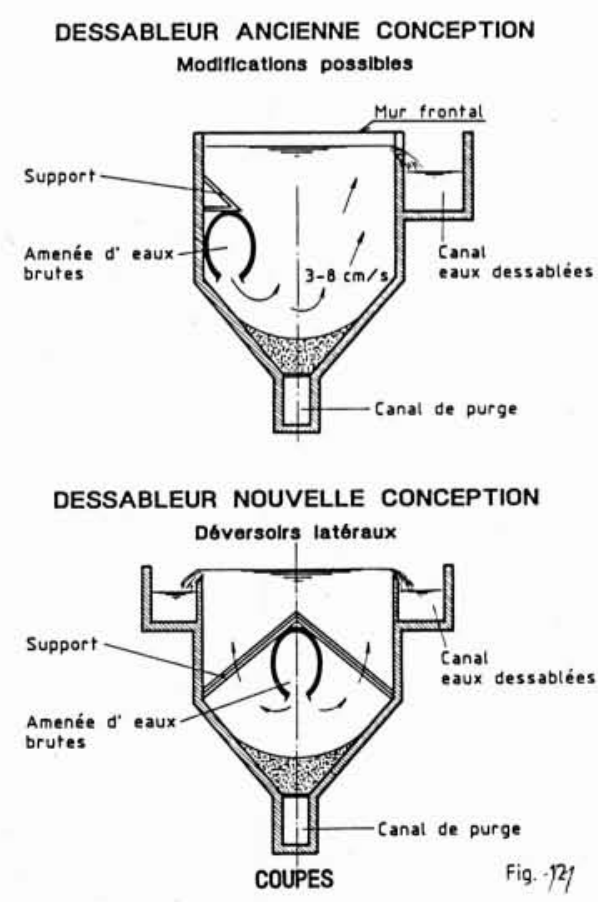

11. Dessableurs du futur. Modification possible et nouvelle conception. 


\subsection{Nouvelle conception de dessableur}

Ainsi, pour des nouveaux ouvrages de dessablage, il est nécessaire de :

- diminuer les hauteurs de décantation, soit la profondeur de l'ouvrage et d'injecter les eaux brutes le plus bas possible!

- diminuer les vitesses moyennes de transit, c'est-à-dire d'avoir des écoulements transversaux au travers d'ouvrages d'une certaine longueur minimale :

- diminuer les effets de la remise en vitesse dans la zone en amont du déversoir, c'est-à-dire d'avoir un déversoir très long.

Pour obtenir ces résultats, il est nécessaire de prévoir :

- un ouvrage long, peu profond, avec une largeur comparable aux ouvrages classiques ;

- l'injection longitudinale ou latérale de l'eau brute par un canal bas placé ;

- un ou des déversoirs latéraux pour avoir de faibles remises en vitesse.

\subsection{Dessableurs du futur: coupes, type avec écoulements transverses (fig. 11)}

Coupe d'un dessableur actuel avec les améliorations possibles à y apporter pour augmenter son efficacité (passage d'un écoulement longitudinal en écoulements transverses !): obturation de l'ancien déversoir et création d'un nouveau déversoir longitudinal, avec un nouveau canal de captage ; injection longitudinale de l'eau brute par un canal répartiteur dans la zone inférieure du dessableur.

Coupe d'un dessableur: nouvelle conception, projetée avec double déversoir longitudinal.

\subsection{Remarques (fig. 12)}

Avec l'application des principes énoncés ci-avant, l'on doit arriver à décanter des sédiments de granulométrie très fine, estimée à $<80 \mu \mathrm{m}$.

En cas de difficultés de construire un canal extérieur de récupération (dessableur en souterrain!), l'on peut prévoir un nouveau canal longitudinal à l'intérieur du dessableur !

Avec un dessableur modifié, l'on peut, à qualité semblable et actuelle de dessablage, tolérer une augmentation de débit, ce qui peut être intéressant lors de transformations d'anciennes installations hydro-électriques.

Sur modèle réduit, l'on a essayé un double fond " rainuré " (type Labaye), posé dans un dessableur de forme classique (voir figure 6, coupe A-A). Cela permet d'améliorer l'efficacité de dessablage. Mais ce double fond doit être conçu avec une forte pente et avec une large cheminée de descente des particules.

Cette solution peut s'appliquer aussi bien à un dessableur traditionnel que sur un nouveau dessableur.
COURBES GRANULOMETRIQUES DES MATERIAUX DEPOSES

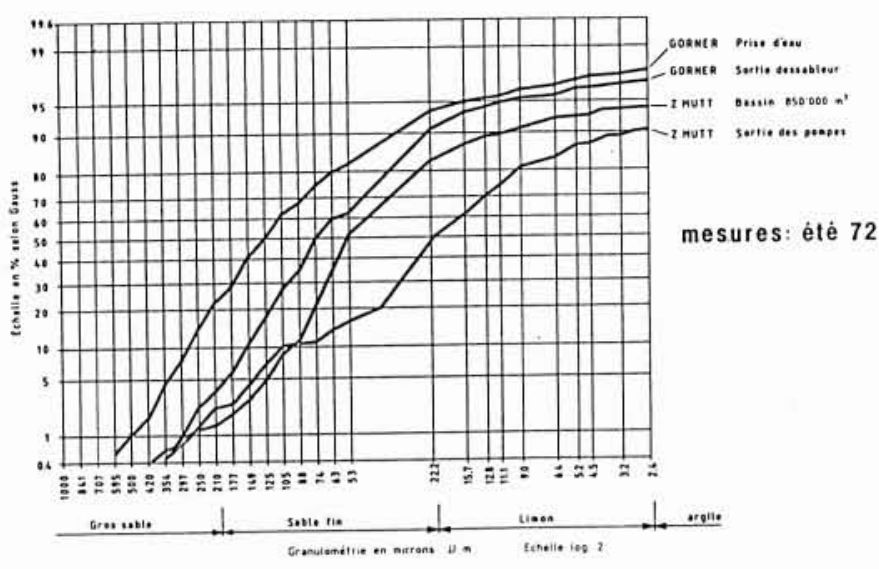

12. Courbes gramulométriques des sédiments après différents

\section{Références}

Bezinge A., F. Schafer, Pompes d'accumulation et eaux glaciaires. Bulletin technique de la Suisse romande, $49^{\mathrm{e}}$ année, $\mathrm{n}^{\circ} 20$ : 1968.

Bezinge A., F. Schafer, Storage pumps and glacial waters. The british hydromechanics research association-September 1969.

Bezinge A., R. Aeschlimann, 1977, Forces Motrices de Conches S.A. Aménagement hydro-électrique du Fieschertal, GKW III. Rapport: buts et description des ouvrages définitifs. Conception et essais sur modèle réduit. Expériences d'exploitation après 1975 et 1976.

BEZINGE A., Torrents glaciaires, Hydrologie et chariages d'alluvions. Société Suisse des Sciences Naturelles; $5-8$ octobre 1978, Brigue.

Bezinge A., G. Bonvin, Bassin de compensation de Z'Mutt et sédiments. Symposium international sur la sédimentaiton de retenues en rivières et de réservoirs dans le secteur alpin les 22 et 23 octobre 1981 à Zurich.

Bezinge A., R. Aeschlimann, Rehaussement de la prise de vidange de fond du barrage de Grande Dixence. Symposium international sur la sédimentation des retenues. VAW - ETH Zurich, les 22-23 octobre 1981.

Bezinge A., P. Aasheim, Ph. Hertig, Prise d'eau flottante pour le pompage d'eaux glaciaires. Bulletin technique des Ateliers de Constructions Mécaniques de Vevey, 1985.

BEZINGE A., Enquête et estimations des charriages annuels sur différents bassins versants du Rhône. Mars 1985. Expertise non publiée.

BEZINGE A., Dangers d'exploitation d'ouvrages hydrauliques. Expériences réalisées à Grande Dixence. Journée technique du 20 avril 1986 de l'Association valaisanne des producteurs d'énergie électrique.

Bezinge A., R. Aeschlimann, 30 ans d'expériences sur les captages d'eaux glaciaires de Grande Dixence. Journée technique du 27 novembre 1987 de l'Association valaisanne des producteurs d'énergie électrique. 
Bezinge A., R. Aeschlimann, Lutte contre les usures sur les ouvrages hydrauliques de Grande Dixence. Symposium érosion, abrasion et cavitation en ouvrages hydrauliques. VAW - ETH Zurich, 19-21 octobre 1988.

Bezinge A., M. J. Clark, A. M. Gurnell and J. Warburton, The management of sediment transported by glacial meltwater streams and its significances for the study of sediment transport processes. Symposium de la société Internationale de glaciologie, Norvège, octobre 1988.

Bezinger A. Glacier de Findelen. Avance rapide et construction d'un nouveau captage sous-glaciaire. Revue LA Houllle BLANCHE N 6/7-1984.

SChafer F., A. Bezinge, Alpine Kraftwerke, Speicherpumpen, Triebwasserqualität, Erosion und Werkstoffe. Pumpentagung VDMA, Karlsruhe, Okt. 1988.

Shafer F., A. Bezinge, Auswirken einer Schwimmenden Wasserfassung auf den Verschleiss von zentrifugal pumpen. VAW ETH Zurich, 19-21 Okt. 1988.
BENSON C., W. HARRISON et autres, Geophysical Institut University of Alaska, Fairbank, June 1986. Final Report, Workshop on Alaska Hydrology : problemes related to glacierized basins (comme participant!)

SHF, $2^{\mathrm{e}}$ journées de l'hydraulique, Grenoble, 25-29 juin 1952. Transports hydrauliques et décantation des matériaux solides.

BOVET Th., 'Contribution à l'étude du phénomène d'érosion par frottament dans les turbines hydrauliques. Bulletin technique de la suisse romande $3: 1958$.

Tsuguo NOZAKI, Nuevo metodo para la decision de la capacidad y dimension del desarenador, considerando el desgaste de la turbina por el material solido flotante. Japan International Cooperation Agency, enero 1985.

Collıns D. N., Université de Manchester. Département de géographie. Alpine Glacier Project: working Paper Series 1977-1988.

Gurnell A. M., M. J. Clark, Glacio-fluvial sediment transfer an alpine perspective, 1987. Edit. Chichester: John Wiley, Symposium Erosion. 